Publ. RIMS, Kyoto Univ.

14 (1978), 53-69

\title{
On Limiting Gibbs States of the Two-Dimensional Ising Models
}

\author{
By \\ Yasunari HIGUCHI*
}

\begin{abstract}
We consider limiting Gibbs states in the two-dimensional ferromagnetic Ising model at sufficiently low temperatures. We prove that every limiting Gibbs state corresponding to a boundary condition such that $N^{+} / N^{-}<\theta<3 / 5$ on every boundary is $/ 1^{-}$, where $N^{+}$ is the number of up-spins on the boundary and $N^{-}$is that of down-spins. We also prove that for each $\theta>3 / 5$, there exists a boundary condition such that $3 / 5<N^{+} / N^{-} \leq \theta$ on every boundary, and the limiting Gibbs state corresponding to this boundary condition is $\mu^{+}$.
\end{abstract}

\section{$\S$ 1. Introduction}

It is conjectured in general that every Gibbs state is a convex combination of $\mu^{+}$and $\mu^{-}$in the two-dimensional ferromagnetic Ising models while Dobrushin [1] has shown that it is not true in the three-dimensional case. Indeed, this conjecture was proved for translationally invariant Gibbs states by Gallavotti and Miracle-Sole [3] (at low temperatures) and by Messager and Miracle-Sole [6] (up to the critical temperature). The uniqueness of the Gibbs state at temperatures not lower than the critical one was shown by Lebowitz and Martin-Löf [5].

In order to prove the above conjecture, it is enough to show it for the limiting Gibbs states. Gallavotti [2] showed that the boundary condition with up-spins in the upper half plane and down-spins in the lower half plane gives us $\left(\mu^{r}+\mu^{-}\right) / 2$ as the limiting Gibbs state at low temperatures. Recently Abraham and Reed [9] proved that the above boundary condition shifted upwards by $\alpha \sqrt{2 n}$, where $2 \mathrm{n}$ is the length of the side of the $\mathrm{n}$-th box, gives us the limiting Gibbs state $C_{\alpha} \mu^{+}+(1$ $\left.-\mathrm{C}_{\alpha}\right) \mu^{-}\left(\mathrm{C}_{\alpha}\right.$ is exactly given $)$ at any temperature lower than the critical

Communicated by K. Itô, February 4, 1977.

* Department of Mathematics, Kyoto University, Kyoto 606, Japan.

Present Address: Department of Mathematics, Kobe University, Kobe 657, Japan. 
one. Also at the symposium in Oberwolfach in October 1976, Messager and Miracle-Sole reported that the boundary condition with up-spins in the upper half plane and arbitrary spins in the lower one gives us a translationally invariant limiting Gibbs state.

In this paper, we will prove what we stated in the abstract. We will also show that if the boundary condition is periodic on every boundary with a period independent of the size of the box, and if $N^{-} / N^{-} \leq \theta<1$ on every boundary, then we have $\mu^{-}$as the limiting Gibbs state.

We will state our results in $\S 2$ with the necessary preparation of notations which are used throughout the paper. In $\S 3$ we will show a key lemma for the proof of the results. The proofs of our theorems are given in $\S 4$ and $\S 5$.

\section{$\S 2 . \quad$ Results}

Let $Z^{2}$ be the two-dimensional lattice and $\Omega=\{+1,-1\}^{Z^{2}}$ be the space of all possible spin configurations on $Z^{2}$. We assign $\Omega$ a product topology. Let $V$ be a finite subset of $Z^{2}$ and $\partial V=\left\{x \in Z^{2} \backslash V ; x\right.$ is a nearest neighbour of $V\}$ its boundary. For each $\omega \in \Omega$, the energy on $V$ with boundary condition $\omega$ is a real valued function $E_{V}^{\omega}(\cdot)$ on $\Omega_{V}$ $=\{+1,-1\}^{v}$ such that

$$
E_{V}^{\omega}(\sigma)=-\beta \sum_{x, y \in V}^{*} \sigma(x) \sigma(y)-\beta \sum_{x \in V, y \equiv \partial V}^{*} \sigma(x) \omega(y),
$$

where $\sum_{x \in A, y \in B}^{*}$ means the summation over all pairs $\{x, y\}$ such that (i) $x \in A, y \in B$ and (ii) $x$ and $y$ are nearest neighbours. The finite Gibbs state on $\Omega_{V}$ with boundary condition $\omega$ is the probability measure defined by

$$
P_{V}^{\omega}(\sigma)=\left[Z_{V}^{\omega}\right]^{-1} \exp \left\{-E_{V}^{\omega}(\sigma)\right\}, \quad \sigma \in \Omega_{V},
$$

where

$$
Z_{V}^{\omega}=\sum_{\sigma=\Omega_{V}} \exp \left\{-E_{V}^{\omega}(\sigma)\right\}
$$

$P_{V}^{\omega}$ is also regarded as a probability measure on $\Omega$ in the usual way.

Throughout this paper we consider

$$
V_{n}=\left\{x=\left(x^{1}, x^{2}\right) \in Z^{2} ;-n+1 \leq x^{i} \leq n, i=1,2\right\}
$$

and denote $P_{n}^{\omega}=P_{V_{n}}^{\omega}, \Omega_{n}=\Omega_{V_{n}}$ for simplicity. We put $\partial V_{n}=V_{n+1} \backslash V_{n}$ 
which does not change the definition of $P_{n}^{\omega}$. Our problem is to study the limiting measure of $P_{n}^{\omega}$ as $n \rightarrow \infty$, i.e. the limiting Gibbs states. We denote by $\mu^{+}$and $\mu^{-}$the limiting Gibbs states corresponding to the boundary conditions $\omega^{+}$and $\omega^{-}$respectively, where $\omega^{+}(x)=+1$ and $\omega^{-}(x)=-1$ for all $x \in Z^{2}$.

Our results about the limiting Gibbs states are the followings.

Theorem 1. For any $\delta<3$, there exists $\beta_{1}(\delta) \in(0, \infty)$ such that for any $\omega \in \Omega$ with

$$
\lim _{n \rightarrow \infty} \frac{1}{n} \sum_{x \in \partial V_{n}} \max \{\omega(x), 0\} \leq 0,
$$

and for any $\beta>\beta_{1}(\delta), P_{n}^{\omega}$ converges weakly to $\mu^{-}$as $n \rightarrow \infty$.

Theorem 2. For any $3<\delta<4$, there exists some $\beta_{2}(\delta) \in(0, \infty)$ and $\omega \in \Omega$ such that

$$
\lim _{n \rightarrow \infty} \frac{1}{n} \sum_{x \in \partial V_{n}} \max \{\omega(x), 0\}=\hat{0},
$$

and $P_{n}^{\prime \prime}$ converges weakly to $\mu^{+}$as $n \rightarrow \infty$ for $\beta>\beta_{2}(\delta)$.

Theorems 1 and 2 imply that $\delta=3$ is critical for this convergence. However for special $\omega \in \Omega$, we have a sharper result. To state it, we prepare

Definition 1 . Let $p$ be a positive integer and $\bar{\omega} \in\{+1,-1\}^{p}$. We say that $\omega \in \Omega$ is periodic on $\partial V_{n}$ with the unit configuration $\bar{\omega}$ if $\bar{\omega}$ appears repeatedly on the boundary $\partial V_{n}$ clockwise. There may remain less than $P$ points on $\partial V_{n}$, on which we let $\omega$ to be arbitrary (see Fig. 1).

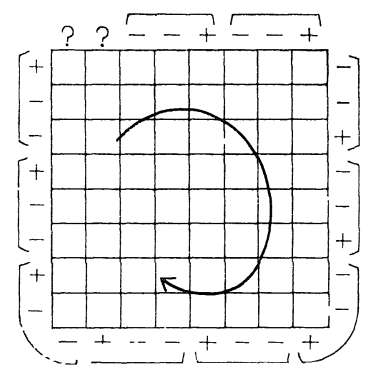

Fig. 1. Example of periodic boundary condition $\bar{\omega}=\{-,-,+\} \quad(p=3)$ 
Theorem 3. For any $0<\delta<4$, there exists $\beta_{3}(\delta) \in(0, \infty)$ such that for any $\beta>\beta_{3}(\delta), P_{n}^{\omega}$ converges weakly to $\mu^{-}$as $n \rightarrow \infty$, if $\omega$ is periodic with the unit $\bar{\omega}$ on every boundary for some $\bar{\omega} \in\{+1,-1\}^{p}$ with integer $p>0$, and if

$$
\varlimsup_{n \rightarrow \infty} \frac{1}{n} \sum_{x \in \partial V_{n}} \max \{\omega(x), 0\} \leq \sigma .
$$

\section{§ 3. Fundamental Lemma}

To prove the theorems in the preceding section, we apply so called "the contour method." For $\sigma \in \Omega_{n}, \omega \in \Omega$, we associate the pair $\left(\sigma,\left.\omega\right|_{\partial V_{n}}\right)$ with a set of connected bonds of dual lattice $L$ of $Z^{2}$, which gives the separating lines between the regions of opposite spins (see Fig. 2).

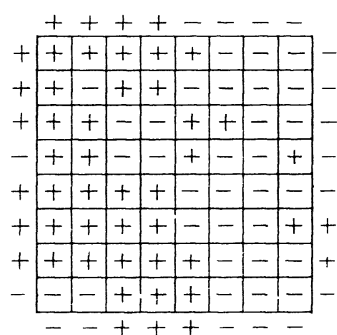

(a) $\left(\sigma,\left.\omega\right|_{\partial V_{n}}\right)$

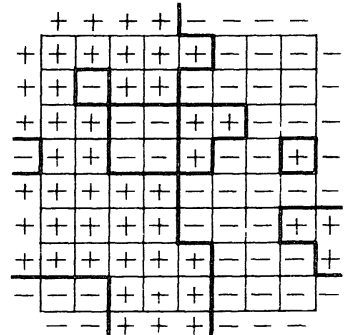

(b) separating lines

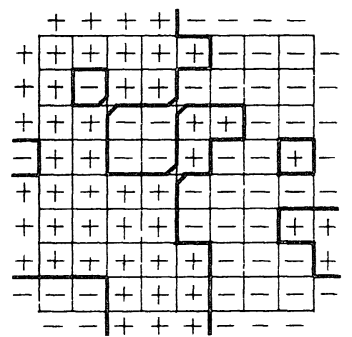

(c) contours

Fig. 2 .

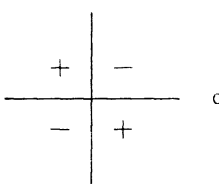

(a)

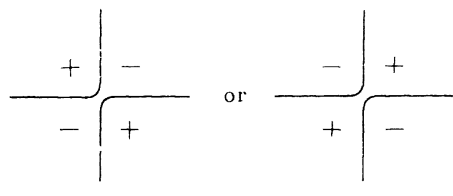

(b)

Fig. 3.

These separating lines form some connected graphs in $L$. When a vertex of $L$ belongs to four bonds contained in one of the above graphs, we separate this graph at this point as in Fig. 3. Then we obtain a collection $C_{n}(\sigma, \omega)$ of contours in

$$
U_{n}=\left\{\left(u^{1}, u^{2}\right) \in L ;-\mathrm{n}-1 / 2 \leq u^{i} \leq n+3 / 2, i=1,2\right\}
$$


(see Fig. 2). Thus we have a one-to-one map from $\Omega_{n}$ into the set of all collections of contours in $U_{n}$ for each fixed $\omega$. There may appear two types of contours: one is closed and the other is open. Any open contour starts from some point of $U_{n} \backslash U_{n-1}$ and ends at another point of $U_{n} \backslash U_{n-1}$. We denote the collection of closed contours in $C_{n}(\sigma, \omega)$ by $\Gamma_{n}(\sigma, \omega)=\left\{\gamma_{n}^{1}(\sigma, \omega), \cdots, \gamma_{n}^{s}(\sigma, \omega)\right\}$ and the collection of open contours by $\Lambda_{n}(\sigma, \omega)=\left\{\lambda_{n}^{1}(\sigma, \omega), \cdots, \lambda_{n}^{t}(\sigma, \omega)\right\}$, where $s$ depends on $n, \sigma$ and $\omega$, and $t$ depends on $n$ and $\omega$. Then it is easy to see that

$$
P_{n}^{\omega}(\sigma)=\left[\widetilde{Z}_{n}^{\omega}\right]^{-1} \exp \left\{-2 \beta\left|\Lambda_{n}(\sigma, \omega)\right|-2 \beta\left|\Gamma_{n}(\sigma,(1))\right|\right\}
$$

where

$$
\left|\Gamma_{n}(\sigma, \omega)\right|=\sum_{i=1}^{s}\left|\gamma_{n}^{i}(\sigma, \omega)\right| \text { and }\left|\Lambda_{n}(\sigma, \omega)\right|=\sum_{i=1}^{t}\left|\lambda_{n}^{i}(\sigma, \omega)\right|
$$

are the total lengths of closed and open contours in $C_{n}(\sigma, \omega)$ respectively, and

$$
\tilde{Z}_{n}^{\omega}=\sum_{\sigma \in \Omega_{n}} \exp \left\{-2 \beta\left|\Lambda_{n}(\sigma, \omega)\right|-2 \beta\left|\Gamma_{n}(\sigma, \omega)\right|\right\}
$$

Define $\mathcal{O}_{n}(\omega)=\left\{\Lambda_{n}(\sigma, \omega) ; \sigma \in \Omega_{n}\right\}$ and $m_{n}(\omega)=\min \left\{|\Lambda| ; \Lambda \in \mathcal{O}_{n}(\mathcal{}(\omega)\}\right.$.

Note that

$$
m_{n}(\omega) \leq \min \left\{N_{n}^{+}(\omega), N_{n}^{-}(\omega)\right\} \leq 4 n
$$

where

$$
N_{n}^{+}(\omega)=\sum_{x \in \partial V_{n}} \max \{\omega(x), 0\} \quad \text { and } \quad N_{n}^{-}(\omega)=\sum_{x \in \partial V_{n}} \max \{-\omega(x), 0\} .
$$

Lemma 1. For any $\varepsilon>0$, if $\beta>\beta(\varepsilon)=\frac{4+\varepsilon}{2 \varepsilon} \log 3$,

$$
\lim _{n \rightarrow \infty} P_{n}^{\omega}\left(\left\{\sigma \in \Omega_{n} ;\left|\Lambda_{n}(\sigma, \omega)\right| \geq m_{n}(\omega)+\varepsilon n\right\}\right)=0
$$

for any $\omega \in \Omega$.

Proof. Let us fix $\omega \in \Omega, n>1$, and $\Lambda \in \mathcal{O}_{n}(\omega)$ arbitrarily. First, we will estimate the probability of the set $\left\{\sigma \in \Omega_{n} ; \Lambda_{n}(\sigma, \omega)=\Lambda\right\}$. Take any $\Lambda^{*} \in \mathcal{O}_{n}(\omega)$ such that $\left|\Lambda^{*}\right|=m_{n}(\omega)$. Let $\sigma_{\tilde{\Lambda}}$ be the element of $\Omega_{n}$ such that (i) $\Lambda_{n}\left(\sigma_{\tilde{A}}, \omega\right)=\tilde{\Lambda}$, (ii) $\Gamma_{n}\left(\sigma_{\tilde{\Lambda}}, \omega\right)=\phi, \tilde{\Lambda}=\Lambda, \Lambda^{*}$. Then we can define a bijection $T_{A A^{*}}: \Omega_{n} \rightarrow \Omega_{n}$ by 


$$
\left(T_{\Lambda \Lambda^{*}} \sigma\right)(x)=\left\{\begin{aligned}
\sigma(x) & \text { if } x \notin D\left(\Lambda, \Lambda^{*}\right), \\
-\sigma(x) & \text { if } x \in D\left(\Lambda, \Lambda^{*}\right),
\end{aligned}\right.
$$

where $D\left(\Lambda, \Lambda^{*}\right)=\left\{x \in V_{n} ; \sigma_{\Lambda}(x) \neq \sigma_{\Lambda^{*}}(x)\right\}$.

Note that

$$
\left|\Lambda_{n}\left(T_{\Lambda \Lambda^{*}} \sigma, \omega\right)\right|+\left|\Gamma_{n}\left(T_{\Lambda \Lambda^{*}} \sigma, \omega\right)\right| \leq m_{n}(\omega)+\left|\Gamma_{n}(\sigma, \omega)\right|
$$

for any $\sigma \in \Omega_{n}$ such that $\Lambda_{n}(\sigma, \omega)=\Lambda$. Hence we have

$$
\begin{aligned}
& P_{n}^{\omega}\left(\left\{\sigma \in \Omega_{n} ; \Lambda_{n}(\sigma, \omega)=\Lambda\right\}\right) \\
& \leq e^{-2 \beta\left(|\Lambda|-m_{n}(\omega)\right)}\left[\widetilde{Z}_{n}^{\omega}\right]^{-1} \\
& \quad \times \sum_{\sigma \in \Omega_{n} ; \Lambda_{n}(\sigma, \omega)=\Lambda} \exp \left\{-2 \beta\left|\Lambda_{n}\left(T_{\Lambda \Lambda^{*}} \sigma, \omega\right)\right|-2 \beta\left|\Gamma_{n}\left(T_{\Lambda \Lambda^{*}} \sigma, \omega\right)\right|\right\} \\
& \leq e^{-2 \beta\left(|\Lambda|-m_{n}(\omega)\right)} .
\end{aligned}
$$

On the other hand, it is not hard to verify that

$$
\#\left\{\Lambda \in \mathcal{O}_{n}(\omega) ;|\Lambda|=k\right\} \leq 3^{k} .
$$

Hence combining (7) and (8), we have

$$
\begin{aligned}
P_{n}^{\omega}\left(\left\{\sigma \in \Omega_{n} ;\left|\Lambda_{n}(\sigma, \omega)\right| \geq m_{n}(\omega)+\varepsilon n\right\}\right) \\
\quad \leq \text { Const. } \times 3^{m_{n}(\omega)+\varepsilon n} e^{-2 \beta \varepsilon n} \\
\quad \leq \text { Const. } \times 3^{(4+\varepsilon) n} e^{-2 \beta \varepsilon n} \rightarrow 0 \text { as } n \rightarrow \infty,
\end{aligned}
$$

for $\beta>\beta(\varepsilon)=\frac{4+\varepsilon}{2 \varepsilon} \log 3$, which proves the lemma.

\section{$\S 4$. Proof of Theorem 1}

We will begin this section with some definitions. Let $n>0, \omega \in \Omega$ and $\Lambda \in \mathcal{O}_{n}(\omega)$ be given.

Definition 2. We say that $p \in V_{n}$ and $q \in V_{n}$ are $\Lambda$-equivalent if we can draw a curve in $\left\{\left(x^{1}, x^{2}\right) \in \boldsymbol{R}^{2} ;-n-1 / 2 \leq x^{i} \leq n+3 / 2, i=1,2\right\}$ from $p$ to $q$ without crossing $\Lambda$.

Put $R_{\Lambda}(x)=\left\{y \in V_{n} ; y\right.$ is $\Lambda$-equivalent to $\left.x\right\}$ for $x \in V_{n}$. A contour $\lambda \in \Lambda$ is said to be a defining contour of $R_{\Lambda}(x)$ if $\lambda$ separates $R_{\Lambda}(x)$ from a neighbouring region $R_{\Lambda}(y)$.

The boundary $\partial V_{n}$ is decomposed into four natural sides; 


$$
\begin{aligned}
& A_{n}^{1}=\left\{\left(x^{1}, x^{2}\right) \in \partial V_{n} ; x^{1}=n+1\right\}, \quad A_{n}^{2}=\left\{\left(x^{1}, x^{2}\right) \in \partial V_{n} ; x^{2}=-n\right\}, \\
& A_{n}^{3}=\left\{\left(x^{1}, x^{2}\right) \in \partial V_{n} ; x^{1}=-n\right\},
\end{aligned}
$$

and

$$
A_{n}^{4}=\left\{\left(x^{1}, x^{2}\right) \in \partial V_{n}: x^{2}=n+1\right\} .
$$

We denote the origin by 0 .

Definition 3. Let $A$ be a subset of $\partial V_{n}$. We say that $R_{A}(0)$ touches $A$ if there exist $x \in R_{A}(0)$ and $y \in A$ such that (i) $x$ and $y$ are nearest neighbours and (ii) the bond connecting $x$ and $y$ does not cross $\Lambda$.

Proof of Theorem 1. It is enough to prove the theorem for $2<\delta$ $<3$. Let $\omega \in \Omega$ be a boundary condition satisfying the condition (3). Let us fix $0<\varepsilon<\frac{1}{10}(3-\delta)$ and a sufficiently large $n>0$ such that $N_{n}^{+}(\omega) \leq(\delta+\varepsilon) n$. Take $\beta>\beta(\varepsilon)$, where $\beta(\varepsilon)$ is given in Lemma 1. We divide $\mathcal{O}_{n}(\omega)$ into three parts: $\mathcal{O}_{n}^{1}(\omega)=\left\{\Lambda \in \mathcal{O}_{n}(\omega) ; R_{\Lambda}(0)\right.$ touches only one side of $\left.\partial V_{n}\right\}, \mathcal{O}_{n}^{2}(\omega)=\left\{\Lambda \in \mathcal{O}_{n}(\omega) \backslash \mathcal{O}_{n}^{1}(\omega) ; \Lambda\right.$ has a contour from one side to its opposite side in $\left.U_{n} \backslash U_{n-1}\right\}$ and $\mathcal{O}_{n}^{3}(\omega)=\mathcal{O}_{n}(\omega) \backslash\left\{\mathcal{O}_{n}^{1}(\omega) \cup \mathcal{O}_{n}^{2}(\omega)\right\}$. Then we have

$$
\begin{aligned}
P_{n}^{\omega}(\{\sigma & \left.\left.\in \Omega_{n} ; \sigma(0)=+1\right\}\right) \\
& =\sum_{i=1}^{3} \sum_{\Lambda \in \Theta_{n}^{i}(\omega)} P_{n}^{\omega}\left(\left\{\sigma \in \Omega_{n} ; \sigma(0)=+1, \Lambda_{n}(\sigma, \omega)=\Lambda\right\}\right) .
\end{aligned}
$$

We treat $\mathcal{O}_{n}^{3}(\omega)$ in different ways according to the following two cases of the boundary condition $\omega$. Divide $\partial V_{n}$ into four parts:

$$
\begin{aligned}
& B_{n}^{1}=\left\{\left(x^{1}, x^{2}\right) \in \partial V_{n} ; x^{1}>0, x^{2}>0\right\}, \\
& B_{n}^{2}=\left\{\left(x^{1}, x^{2}\right) \in \partial V_{n} ; x^{1}>0, x^{2} \leq 0\right\}, \\
& B_{n}^{3}=\left\{\left(x^{1}, x^{2}\right) \in \partial V_{n} ; x^{1} \leq 0, x^{2} \leq 0\right\}
\end{aligned}
$$

and

$$
B_{n}^{4}=\left\{\left(x^{1}, x^{2}\right) \in \partial V_{n} ; x^{1} \leq 0, x^{2}>0\right\} .
$$

Boundary conditions are classified into

$$
\text { Case } 1 ; \quad \sum_{x=B_{n}^{i}} \max \{\omega(x), 0\} \leq(2-\varepsilon) n \text { for all } i,
$$


Case $2 ; \quad \sum_{x \in B_{n}^{i}} \max \{\omega(x), 0\}>(2-\varepsilon) n$ for some $i$.

However $\mathcal{O}_{n}^{1}(\omega)$ and $\mathcal{O}_{n}^{2}(\omega)$ are studied in the same way for all $\omega$.

Let us put $\mathcal{L}_{n}=\left\{\Lambda \in \mathcal{O}_{n}(\omega) ;|\Lambda| \geq m_{n}(\omega)+\varepsilon n\right\} . \quad$ By Lemma $1, P_{n}^{\omega}(\{\sigma$ $\left.\left.\in \Omega_{n} ; \Lambda_{n}(\sigma, \omega) \in \mathcal{L}_{n}\right\}\right) \rightarrow 0$ as $n \rightarrow \infty$ for all $\omega$.

Now consider $\Lambda \in \mathcal{O}_{n}^{1}(\omega)$. Then $R_{A}(0)$ touches only one side, say $A_{n}^{1}$. There exists a defining contoun $\lambda$ of $R_{\Lambda}(0)$ form $\left(n+3 / 2, h_{1}\right)$ to $\left(n+3 / 2, h_{2}\right)$ such that $|\lambda| \geq 2 n+\left|h_{1}-h_{2}\right|$. Reversing the spins in $R_{A}(0)$, we obtain the inequality $|\Lambda| \geq m_{n}(\omega)+2 n$. Hence $\Lambda \in \mathcal{L}_{n}$ and so

(9) $\sum_{\Lambda \in O_{n}^{1}(\omega)} P_{n}^{\omega}\left(\left\{\sigma \in \Omega_{n} ; \sigma(0)=+1, \Lambda_{n}(\sigma, \omega)=\Lambda\right\}\right) \rightarrow 0$ as $n \rightarrow \infty$.

Next, consider $\Lambda \in \mathcal{O}_{n}^{2}(\omega)$. If $R_{\Lambda}(0)$ touches the minus boundary, i.e. $R_{A}(0)$ touches the set $\left\{x \in \partial V_{n} ; \omega(x)=-1\right\}$, then putting $D_{A}(0)$ $=R_{\Lambda}(0) \backslash\left\{x \in R_{\Lambda}(0) ; \sigma(\mathrm{x})=-1\right.$ for all $\sigma \in \Omega_{n}$ such that $\left.\Lambda_{n}(\sigma, \omega)=\Lambda\right\}$, we have

$$
\begin{aligned}
& P_{n}^{\omega}\left(\left\{\sigma \in \Omega_{n} ; \sigma(0)=+1, \Lambda_{n}(\sigma, \omega)=\Lambda\right\}\right) \\
& \quad=P_{n}^{\omega}\left(\left\{\sigma \in \Omega_{n} ; \Lambda_{n}(\sigma, \omega)=\Lambda\right\}\right) P_{D_{\Lambda}(0)}^{\omega^{-}}(\{\sigma ; \sigma(0)=+1\}) \\
& \quad \leq P_{n}^{\omega}\left(\left\{\sigma \in \Omega_{n} ; \Lambda_{n}(\sigma, \omega)=\Lambda\right\}\right) P_{n}^{\omega-}(\{\sigma ; \sigma(0)=+1\})
\end{aligned}
$$

from FKG inequality.* We will show that $\Lambda \in \mathcal{L}_{n}$ if $R_{\Lambda}(0)$ touches the plus boundary. Since $\Lambda \in \mathcal{O}_{n}^{2}(\omega)$, there exists a contour $\lambda$ from one side to the opposite side of $U_{n} \backslash U_{n-1}$. If $\Lambda$ has more than one such contours, then $|\Lambda| \geq 4 n \geq N_{n}^{+}(\omega)+n \geq m_{n}(\omega)+n$ and so $\Lambda \in \mathcal{L}_{n}$. So we can assume that $\Lambda$ has only one such contour $\lambda$. We can also assume without loss of generality that $\lambda$ is a contour from $\left(n+\frac{3}{2}, h_{1}\right)$ to $\left(-n-\frac{1}{2}, h_{2}\right)$ such that the origin lies below $\lambda$. In this case, note that

$$
|\Lambda| \geq|\lambda|+\sum_{x \in \partial V_{n}^{\prime}} \max \{\omega(x), 0\}+\sum_{x \in \partial V_{n^{\prime \prime}}} \max \{-\omega(x), 0\}
$$

if $\omega\left(x^{\prime}\right)=-1$ and $\omega\left(x^{\prime \prime}\right)=+1$, and

$$
|\Lambda| \geq|\lambda|+\sum_{x \in \partial V_{n^{\prime}}} \max \{-\omega(x), 0\}+\sum_{x \in \partial V_{n^{\prime \prime}}} \max \{\omega(x), 0\}
$$

if $\omega\left(x^{\prime}\right)=+1$ and $\omega\left(x^{\prime \prime}\right)=-1$, where $\partial V_{n}^{\prime}=\left\{x \in \partial V_{n} ; x\right.$ lies above $\left.\lambda\right\}$ $\partial V_{n}^{\prime \prime}=\left\{x \in \partial V_{n} ; x\right.$ lies below $\left.\lambda\right\}$ and $x^{\prime}, x^{\prime \prime}$ are the points such that (i) $x^{\prime} \in \partial V_{n}^{\prime}$ and $x^{\prime \prime} \in \partial V_{n}^{\prime \prime}$, (ii) $x^{\prime}$ and $x^{\prime \prime}$ are nearest neighbours. If

\footnotetext{
* See e.g. [10].
} 
$\lambda$ is not a defining contour of $R_{\Lambda}(0)$, then $|\Lambda| \geq 4 n$ and so $\Lambda \in \mathcal{L}_{n}$. If $\lambda$ is a defining contour of $R_{1}(0)$ then $\omega\left(x^{\prime}\right)=-1$ and $\omega\left(x^{\prime \prime}\right)=+1$ since $R_{\Lambda}(0)$ touches the plus boundary. It is easy to see that $|\lambda| \geq 2 n+\left(h_{1}^{-}\right.$ $\left.+h_{2}^{-}\right)$and $\sum_{x \in \overrightarrow{\partial V}_{n^{\prime \prime}}} \max \{-\omega(x), 0\} \geq 4 n+h_{1}+h_{2}-N_{n}^{+}(\omega)$, where $h^{-}=\max$ $\{-h, 0\}$. Hence $|A| \geq 6 n-N_{n}^{+}\left((\omega) \geq 6 n-(\delta+\varepsilon) n \geq m_{n}(\omega)+9 \varepsilon n\right.$ and so $A$ $\in \mathcal{L}_{n}$. Thus, we obtain

$$
\begin{aligned}
& \sum_{\Lambda \in \mathcal{O}_{n}^{2}(\omega)} P_{n}^{\omega}\left(\left\{\sigma \in \Omega_{n} ; \sigma(0)=+1, \Lambda_{n}(\sigma,(1))=\Lambda\right\}\right) \\
& \quad \leq P_{n}^{\omega}\left(\left\{\sigma \in \Omega_{n}: \Lambda_{n}(\sigma,(1)=\Lambda\}\right) P_{n}^{\omega^{-}}\left(\left\{\sigma \in \Omega_{n} ; \sigma(0)=+1\right\}\right)+o(1)\right.
\end{aligned}
$$

as $n \rightarrow \infty$.

Now we consider $\mathcal{O}_{n}^{3}(\omega)$ in two cases.

Case 1: $\sum_{x \in B_{n}^{i}} \max \{\omega(x), 0\} \leq(2-\varepsilon) n$ for all $i$. If $\Lambda \in \mathcal{O}_{n}^{3}(\omega)$, $R_{A}(0)$ touches two or four sides of $\partial V_{n}$. Assume that $R_{A}(0)$ touches the plus boundary. If $R_{\Lambda}(0)$ touches four sides of $\partial V_{n}$, we have $|\Lambda|$ $\geq N_{n}^{-}(\omega)=8 n-N_{n}^{+}(\omega) \geq 5 n \geq m_{n}(\omega)+2 n$ and so $\Lambda \in \mathcal{L}_{n}$. If $R_{\Lambda}(0)$ touches two sides of $\partial V_{n}$, say $A_{n}^{1}$ and $A_{n}^{2}$, then there exists a contour $\lambda \in A$ from $\left(n+\frac{3}{2}, h_{1}\right)$ to $\left(h_{2},-n-\frac{1}{2}\right)$ such that the origin lies below $\lambda$. where $\left|h_{1}\right|,\left|h_{2}\right|<n$. Then it is easy to see that $|\lambda| \geq 2 n+\left|h_{1}\right|+\left|h_{2}\right|$ and $|\Lambda \backslash\{\lambda\}| \geq\left(\varepsilon n-h_{1}^{-}-h_{2}^{-}\right)^{\vdash}+N_{n}^{+}(\omega)-\left(2 n+h_{1}-h_{2}-\left(\varepsilon n-h_{1}^{-}-h_{2}^{+}\right)^{-}\right)$, where $h^{+}=\max \{h, 0\}$ and $h^{-}=\max \{-h, 0\}$. Therefore we have

$$
|\Lambda| \geq N_{n}^{+}(\omega)+2\left(\varepsilon n-h_{1}^{-}-h_{2}^{+}\right)^{+}+2\left(h_{1}^{-}+h_{2}^{+}\right) \geq m_{n}(\omega)+2 \varepsilon n
$$

and so $\Lambda \in \mathcal{L}_{n}$. If $R_{A}(0)$ touches the minus boundary, then the same argument as the one showing (10) gives us

$$
\begin{aligned}
& P_{n}^{\omega}\left(\left\{\sigma \in \Omega_{n} ; \sigma(0)=+1, \Lambda_{n}(\sigma, \omega)=\Lambda\right\}\right) \\
& \quad \leq P_{n}^{\omega}\left(\left\{\sigma \in \Omega_{n} ; \Lambda_{n}(\sigma, \omega)=\Lambda\right\}\right) P_{n}^{\omega^{-}}\left(\left\{\sigma \in \Omega_{n} ; \sigma(0)=+1\right\}\right) .
\end{aligned}
$$

Thus we obtain

$$
\begin{aligned}
\sum_{\Lambda \in \mathcal{O}^{3}(\omega)} P_{n}^{\omega}( & \left.\left\{\sigma \in \Omega_{n} ; \sigma(0)=+1, \Lambda_{n}(\sigma, \omega)=\Lambda\right\}\right) \\
\leq & P_{n}^{\omega}\left(\left\{\sigma \in \Omega_{n} ; \Lambda_{n}(\sigma, \omega) \in \mathcal{O}_{n}^{3}(\omega)\right\}\right) \\
& \times P_{n}^{\omega^{-}}\left(\left\{\sigma \in \Omega_{n} ; \sigma(0)=+1\right\}\right)+o(1)
\end{aligned}
$$

as $n \rightarrow \infty$.

Let us take the subsequence $\left\{n^{\prime}\right\}$ such that $\omega$ satisfies the condition of 
case 1 for each $n^{\prime}$. Then combining (9), (11) and (12) we have

$$
\varlimsup_{n^{\prime} \rightarrow \infty} P_{n^{\prime}}^{\omega}\left(\left\{\sigma \in \Omega_{n} ; \sigma(0)=+1\right\}\right) \leq \mu^{-}(\{\sigma \in \Omega ; \sigma(0)=+1\}),
$$

which implies

$$
\lim _{n^{\prime} \rightarrow \infty} P_{n^{\prime}}^{n}\left(\left\{\sigma \in \Omega_{n} ; \sigma(0)=+1\right\}\right)=\mu^{-}(\{\sigma \in \Omega ; \sigma(0)=+1\}) .
$$

Case 2: $\sum_{x \in B_{n}^{i}} \max \{\omega(x), 0\}>(2-\varepsilon) n$ for some $i$. We assume $i=1$. Define $\widetilde{B}_{n}^{1}=\left\{\left(x^{1}, x^{2}\right) \in \partial V_{n} ; x^{1}, x^{2} \geq-(\delta+3 \varepsilon-2) n\right\} \supset B_{n}^{1}$. Let $\Lambda \in \mathcal{O}_{n}^{3}(\omega)$ If $\Lambda$ has a contour from $A_{n}^{1} \backslash \widetilde{B}_{n}^{1}$ (or $A_{n}^{4} \backslash \widetilde{B}_{n}^{1}$ ) to $A_{n}^{4} \backslash B_{n}^{1}$ (or $A_{n}^{1} \backslash B_{n}^{1}$ respectively), then $|\Lambda| \geq(\delta+3 \varepsilon) n \geq N_{n}^{+}(\omega)+2 \varepsilon n \geq m_{n}(\omega)+2 \varepsilon n$ and so $\Lambda \in \mathcal{L}_{n}$. Even if $\Lambda$ has a contour from $\partial V_{n} \backslash \widetilde{B}_{n}^{1}$ to $B_{n}^{1}$ we have

$$
|\Lambda| \geq(\delta+3 \varepsilon-2+2-\varepsilon) n \geq N_{n}^{+}(\omega)+\varepsilon n \geq m_{n}(\omega)+\varepsilon n
$$

and so $\Lambda \in \mathcal{L}_{n}$. Remove the above two kinds of $\Lambda$ 's from $\mathcal{O}_{n}^{3}(\omega)$ and denote the remainder by $\mathscr{Q}_{n}(\omega)$. For $\Lambda \in \mathscr{L}_{n}(\omega)$, let $\tilde{\Lambda}$ be the collection of all contours in $\Lambda$ starting from $\partial V_{n} \backslash \widetilde{B}_{n}^{1}$ and $\widetilde{\mathscr{L}}_{n}(\omega)=\left\{\widetilde{\Lambda} ; \Lambda \in \mathscr{L}_{n}(\omega)\right\}$. Take $\tilde{\Lambda} \in \widetilde{\mathscr{Q}}_{n}(\omega)$. If $R_{\tilde{\Lambda}}(0)$ touches the set $\left\{x \in \partial V_{n} \backslash B_{n}^{1} ; \omega(x)=+1\right\}$, then (i) $\tilde{\Lambda}$ has a contour $\lambda_{0}$ from $A_{n}^{3}$ to $A_{n}^{2}$ such that the origin lies below $\lambda$, or (ii) $\tilde{\Lambda}$ has not such contours. Estimate $\left|\lambda_{0}\right|$ in the case of (i) and estimate the length of contours from $A_{n}^{2} \cup A_{n}^{3}$ to $A_{n}^{2} \cup A_{n}^{3}$ in the case of (ii). Then we have

$$
|\Lambda| \geq 4 n-\left(N_{n}^{+}(\omega)-(2-\varepsilon) n\right) \geq 3 n \geq m_{n}(\omega)+9 \varepsilon n
$$

and so $\Lambda \in \mathcal{L}_{n}$. Thus, we obtain

$$
\begin{aligned}
\sum_{\Lambda \in O_{n}^{3}(\omega)} & P_{n}^{\omega}\left(\left\{\sigma \in \Omega_{n} ; \sigma(0)=+1, \Lambda_{n}(\sigma, \omega)=\Lambda\right\}\right) \\
\quad & \sum_{\tilde{A} \in \widetilde{\mathscr{P}}_{n}(\omega)} P_{n}^{\omega}\left(\left\{\sigma \in \Omega_{n} ; \sigma(0)=+1, \widetilde{\Lambda}_{n}(\sigma, \omega)=\widetilde{\Lambda}\right\}\right)+o(1) \\
\quad & \sum_{\tilde{\Lambda} \in \widetilde{\mathscr{P}}_{\tilde{n}}^{-}(\omega)} P_{n}^{\omega}\left(\left\{\sigma \in \Omega_{n} ; \sigma(0)=+1, \widetilde{\Lambda}_{n}(\sigma, \omega)=\widetilde{\Lambda}\right\}\right)+o(1)
\end{aligned}
$$

where $\widetilde{\mathscr{L}}_{n}^{-}(\omega)=\left\{\tilde{\Lambda} \in \mathscr{L}(\omega) ; R_{\tilde{\Lambda}}(0)\right.$ touches the set $\left\{x \in \partial V_{n} \backslash B_{n}^{1} ; \omega(x)\right.$ $=-1\}\}$. For $\tilde{\Lambda} \in \widetilde{\mathscr{P}}_{n}^{-}(\omega)$, we have

$$
\begin{aligned}
& P_{n}^{\omega}\left(\left\{\sigma \in \Omega_{n} ; \sigma(0)=+1, \widetilde{\Lambda}_{n}(\sigma, \omega)=\widetilde{\Lambda}\right\}\right) \\
& \quad=P_{D_{\tilde{\Lambda}^{(0)}}^{\bar{\sigma}}}^{\bar{D}}(\sigma(0)=+1) P_{n}^{\omega}\left(\widetilde{\Lambda}_{n}(\sigma, \omega)=\widetilde{\Lambda}\right)
\end{aligned}
$$

where $\bar{\omega}(x)=\omega(x)$ for $x \in \partial V_{n},=-1$ for $x \in \partial D_{\tilde{\Lambda}}(0) \backslash \partial V_{n}$. Using FKG 
inequality, we have

$$
P_{D_{\tilde{X}}^{\tilde{\omega}}}^{\tilde{\tilde{\omega}}}(\sigma(0)=+1) \leq P_{n}^{\omega(\delta, \varepsilon)}(\sigma(0)=+1)
$$

where

$$
(1)(\delta, \varepsilon)(x)= \begin{cases}+1 & \text { for } x \in \bigcup_{n=1}^{\infty} \widetilde{B}_{n}^{1}, \\ -1 & \text { for } x \notin \bigcup_{n=1}^{\infty} \widetilde{B}_{n}^{1}\end{cases}
$$

Hence we have

$$
\begin{aligned}
\sum_{\Lambda \in \mathcal{O}_{n}^{3}(\omega)} & P_{n}^{\omega}\left(\sigma(0)=+1, \Lambda_{n}(\sigma, \omega)=\Lambda\right) \\
& \leq P_{n}^{\omega(\delta, \varepsilon)}(\sigma(0)=+1) P_{n}^{\omega}\left(\Lambda_{n}(\sigma, \omega) \in \mathcal{O}_{n}^{3}(\omega)\right)+o(1)
\end{aligned}
$$

as $n \rightarrow \infty$, which together with (9) and (11) implies

$$
\begin{aligned}
P_{n}^{\omega}\left(\left\{\sigma \in \Omega_{n} ; \sigma(0)=+1\right\}\right) & \\
\leq & P_{n}^{\omega^{-}}(\sigma(0)=+1) P_{n}^{\omega}\left(\Lambda_{n}(\sigma, \omega) \in \mathcal{O}_{n}^{2}(\omega)\right) \\
& \quad+P_{n}^{\omega(\delta, \varepsilon)}(\sigma(0)=+1) P_{n}^{\omega}\left(\Lambda_{n}(\sigma, \omega) \in \mathcal{O}_{n}^{3}(\omega)\right)+o(1) \\
\leq & P_{n}^{\omega(\delta, \varepsilon)}(\sigma(0)=+1)+o(1),
\end{aligned}
$$

because $P_{n}^{\omega-}(\sigma(0)=+1) \leq P_{n}^{\omega(\delta, \varepsilon)}(\sigma(0)=+1)$.

On the other hand $P_{n}^{\omega(\delta, \varepsilon)}$ converges weakly to $\mu^{-}$as $n \rightarrow \infty$ for sufficiently large $\beta$ (see Appendix). Taking the subsequence $\left\{n^{\prime \prime}\right\}$ such that $\omega$ satisfies the condition of case 2 for each $n^{\prime \prime}$, we have

$$
\varlimsup_{n^{\prime \prime} \rightarrow \infty} P_{n^{\prime \prime}}^{\omega}(\sigma(0)=+1) \leq \mu^{-}(\sigma(0)=+1)
$$

which implies

$$
\lim _{n^{\prime \prime} \rightarrow \infty} P_{n^{\prime \prime}}^{(\prime)}(\sigma(0)=+1)=\mu^{-}(\sigma(0)=+1) .
$$

The above arguments apply to any $x \in Z^{2}$, and hence we obtain

$$
\lim _{n \rightarrow \infty} P_{n}^{\omega}(\sigma(x)=+1)=\mu^{-}(\sigma(x)=+1), x \in Z^{2} .
$$

The weak convergence of $P_{n}^{\omega}$ to $/^{-}$follows from FKG inequality and (15).

\section{§ 5. Proofs of Theorem 2 and Theorem 3}

First, we will prove Theorem 2. Let us fix $3<\delta<4$ and $\beta>\beta(\delta-3)$ 
arbitrarily, where $\beta(\cdot)$ is given in Lemma 1 . Define $\omega$ by $\omega(x)= \begin{cases}+1 & \text { if }-\frac{\delta-3}{2}\left|x^{1}\right| \leq x^{2} \leq 2\left|x^{1}\right|, \\ -1 & \text { otherwise }\end{cases}$

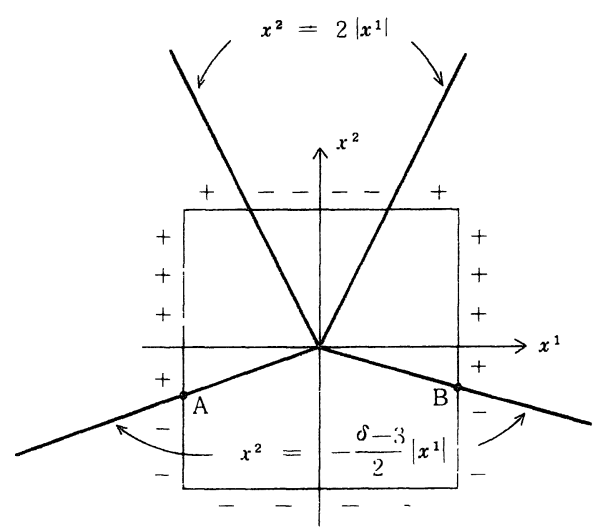

Fig. 4 .

Then it is easy to see that $\lim _{n \rightarrow \infty} \frac{1}{n} N_{n}^{+}(\omega)=\delta$, and $m_{n}(\omega)=3 n$ (see Fig. 4). Let $S_{n}=\left\{\sigma \in \Omega_{n} ; \Lambda_{n}(\sigma, \omega)\right.$ contains contour $\lambda_{A B}(\sigma)$ which connects $A$ and $B\}$. Lemma 1 implies that $P_{n}^{\omega}\left(S_{n}\right) \rightarrow 1$ as $n \rightarrow \infty$. Take $\sigma \in S_{n}$. If $\lambda_{A B}(\sigma)$ intersects the line $l_{-}=\left\{\left(x^{1}, x^{2}\right) \in \boldsymbol{R}^{2} ; x^{1}=0, x^{2} \geq 0\right\}$, then $\left|\lambda_{A B}(\sigma)\right| \geq\{2$ $+(\delta-3)\} n$ and so $\mid \Lambda_{n}(\sigma,(1) \mid \geq \delta n$. Again from Lemma 1 , we have $P_{n}^{\omega}\left(\left\{\sigma \in S_{n} ; \lambda_{A B}(\sigma)\right.\right.$ intersects $\left.\left.l_{+}\right\}\right) \rightarrow 0$

as $n \rightarrow \infty$. Therefore

$$
P_{n}^{\omega}\left(\left\{\sigma \in \Omega_{n} ; R_{A_{n}(\sigma, \omega)}(0) \text { touches the plus boundary }\right\}\right) \rightarrow 1
$$

as $n \rightarrow \infty$. Using FKG inequality we have

$$
P_{n}^{\omega}(\sigma(0)=+1) \geq P_{n}^{\omega^{+}}(\sigma(0)=+1)+o(1)
$$

as $n \rightarrow \infty$. Hence, as in the proof of Theorem 1, we can deduce that $P_{n}^{\omega}$ converges weakly to $\mu^{+}$, which completes the proof of Theorem 2 .

Next we will prove Theorem 3. Fix any $\delta$ such that $0<\delta<4$. Assume that $\omega \in \Omega$ is periodic on every boundary with period $p$ and the 
unit configuration $\bar{\omega} \in\{+1,-1\}^{p}$. It is obvious that

$$
\lim _{n \rightarrow \infty} \frac{1}{n} N_{n}^{+}(\omega)=\frac{8}{p} \sum_{i=1}^{p} \max \{\bar{\omega}(i), 0\} .
$$

We will denote this value by $\bar{\delta}(\bar{\omega})$. The assumption of the theorem is that $\bar{\delta}(\bar{\omega}) \leq \delta$. If $\Lambda \in \mathcal{O}_{n}(\omega)$ has a contour $\lambda$ from one side to the opposite side of $U_{n} \backslash U_{n-1}$, then it is easy to see that $|\Lambda| \geq\left|\Lambda_{n}\left(\omega_{n}^{-}, \omega\right)\right|+2 n \geq m_{n}(1)$ $+2 n$, where $\omega_{n}^{-} \in \Omega_{n}$ is the restriction of $\omega^{-}$to $V_{n}$, i.e. $\omega_{n}^{-}(x)=-1$ for all $x \in V_{n}^{r}$. Hence Lemma 1 implies that

(17) $P_{n}^{\omega}\left(\left\{\sigma \in \Omega_{n} ; \Lambda_{n}(\sigma, \omega)\right.\right.$ has a contour from one side to the opposite side of $\left.\left.U_{n} \backslash U_{n-1}.\right\}\right) \rightarrow 0$

as $n \rightarrow \infty$ for $\beta>\beta(2)$, where $\beta(\cdot)$ is given in Lemma 1 .

From (17) we have

$$
\begin{aligned}
& P_{n}^{\omega}\left(\left\{\sigma \in \Omega_{n} ; R_{A_{n}(\sigma, \omega)}(0) \text { touches the plus boundary }\right\}\right) \\
& \leq P_{n}^{\omega}\left(\left\{\sigma \in \Omega_{n} ;\left|\Lambda_{n}(\sigma, \omega)\right| \geq 8 n-(\delta+\varepsilon) n\right\}\right)+o(1)
\end{aligned}
$$

as $n \rightarrow \infty$ for $\beta>\beta(2)$, where $\varepsilon=\frac{4-\delta}{10}$. Since $\left|\Lambda_{n}\left(\omega_{n}^{-}, \omega\right)\right|=N_{n}^{+}(\omega) \leq(\delta$ $+\varepsilon) n=(4-9 \varepsilon) n$, we have

$$
8 n-(\delta+\varepsilon) n=4 n+9 \varepsilon n>m_{n}(\omega)+9 \varepsilon n .
$$

Then (18) and (19) imply that

$$
P_{n}^{\omega}\left(\left\{\sigma \in \Omega_{n} ; R_{A_{n}(\sigma, \omega)}(0) \text { touches the minus boundary }\right\}\right) \rightarrow 1
$$

as $n \rightarrow \infty$ for $\beta>\beta(9 \varepsilon)=\beta\left(\frac{9}{10}(4-\delta)\right)$. Using the argument as in the proof of Theorem 1, we can prove that $P_{n}^{\omega}$ converges weakly to $\mu^{-}$as $n \rightarrow \infty$ for $\beta>\beta\left(\frac{9}{10}(4-\delta)\right)$. Thus, we have proved Theorem 3 .

\section{Appendix}

In the proof of Theorem 1, we left over the verification of the weak convergence:

$$
\text { (A. 1) } \quad \lim _{n \rightarrow \infty} P_{n}^{\omega(0, \varepsilon)}=\mu^{-}
$$

for $2<\hat{0}<3$ and sufficiently large $\beta>0$. It was told that (A. 1) can be shown by the same idea as in [9]. But for the completeness, we will 
give here a sketch of a proof of (A.1) by our method. We appeal to Gallavotti's formula of representation of $P_{n}^{\omega(\delta, \varepsilon)}$ and estimations of numbers of paths of the random walk. For the simplicity of notations we write $a=\delta-2+3 \varepsilon$ and $\omega=\omega(\delta, \varepsilon)$. Note that $0<a<1$.

Let

$$
W_{n}=\left\{p=\left(p^{1}+\frac{1}{2}, p^{2}+\frac{1}{2}\right) \in L ;\left|p^{i}\right| \leq \log n, i=1,2\right\} .
$$

Any $\Lambda \in \mathcal{O}_{n}(\omega)$ consists of only one contour; $\Lambda=\{\lambda\}$.

Lemma A. 1. $\lim _{n \rightarrow \infty} P_{n}^{\omega}\left(\lambda_{n}(\sigma, \omega) \cap W_{n} \neq \phi\right)=0$.

Proof. We have Gallavotti's formula (cf. [2])

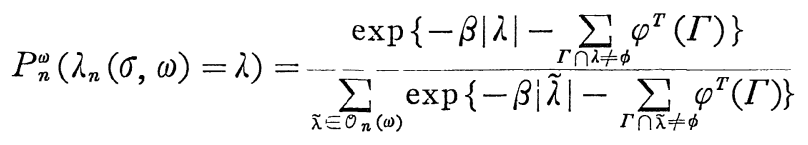

for any $\lambda \in \mathcal{O}_{n}(\omega)$ where $\Gamma$ denotes a finite set of closed contours which are connected with each other. We denote also by $\Gamma$ the union of consisting closed contours as sets in $\boldsymbol{R}^{2}$. The function $\varphi^{T}$ satisfies the estimate*

$$
\sum_{\Gamma \equiv p}\left|\varphi^{T}(\Gamma)\right| \leq \delta(\beta) \rightarrow 0 \quad \text { as } \quad \beta \rightarrow \infty,
$$

for any $p \in L$. Hence we have

$$
\sum_{\Gamma \cap \lambda \neq \phi}\left|\varphi^{T}(\Gamma)\right| \leq \sum_{p \in \lambda} \sum_{\Gamma \ni p}\left|\varphi^{T}(\Gamma)\right| \leq \delta(\beta)|\lambda|
$$

Combining (A. 2) and (A.3) with Lemma 1, we obtain (A. 4)

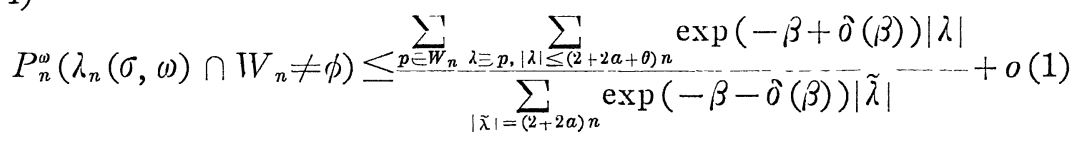

as $n \rightarrow \infty$ for any $\beta>\beta(0)$ where $\theta>0$ is an arbitrarily small constant. The numerator in the right hand side of (A.4) is rewritten into

* The original proof of this estinate in [2] is not correct. But one can get this estimate by careful computation along the same line as that of the original one. There is also another proof by Gallavotti and others in Lecture Notes in Physics, vol. 20, Springer. 


$$
\sum_{p \in W_{n}} \sum_{k=(2+2+2 \alpha) n}^{(2+2 a+\theta) n} R_{n}^{k}(p) \exp (-\beta+\delta(\beta)) k
$$

where $R_{n}^{k}(p)$ is the number of possible contours $\lambda \in \mathcal{O}_{n}(\omega)$ such that $\lambda \ni p$ and $|\lambda|=k$.

It is obvious that $R_{n}^{k}(p)$ is smaller than the number of possible paths of the simple random walk in $L$ which starts from $r_{1}=\left(-a n+\frac{1}{2}\right.$, $\left.n+\frac{3}{2}\right)$ at time 0 , passes through $p$ and ends at $r_{2}=\left(n+\frac{3}{2},-a n+\frac{1}{2}\right)$ at time $k$. Let $T^{d}(q, p)$ denote the number of paths of the random walk which starts from $q=\left(q^{1}+\frac{1}{2}, q^{2}+\frac{1}{2}\right)$ at time 0 and ends at $p=\left(p^{1}+\frac{1}{2}\right.$, $\left.p^{2}+\frac{1}{2}\right)$ at time $d$, where $d-\left|p^{1}-q^{1}\right|-\left|p^{2}-q^{2}\right|$ is non-negative and even. Then we have

$$
R_{n}^{k}(p) \leq \Sigma^{\prime} T^{d_{1}}\left(r_{1}, p\right) T^{d_{2}}\left(r_{2}, p\right)
$$

where $\Sigma^{\prime}$ denotes the summation over all $d_{1}$ and $d_{2}$ such that $d_{1}+d_{2}=k$, $d_{1}-(1+a) n-p^{1}+p^{2}$ and $d_{2}-(1+a) n+p^{1}-p^{2}$ are non-negative and even. On the other hand, we have

$$
\begin{aligned}
T^{d}(q, p) & =\sum^{\prime \prime}\left(\begin{array}{l}
d \\
s_{1}
\end{array}\right)\left(\begin{array}{l}
s_{1} \\
t_{1}
\end{array}\right)\left(\begin{array}{l}
s_{2} \\
t_{2}
\end{array}\right) \\
& \leq \sum_{t_{1} \mid t_{2}=\left(d-m_{1}-m_{2}\right) / 2} \frac{d !}{m_{1} ! m_{2} !\left(t_{1} ! t_{2} !\right)^{2}} \\
& \leq \sum_{t_{1} \cdot t_{2}=\left(d-m_{1}-m_{2}\right) / 2} \frac{d ! 2^{d-m_{1}-m_{2}}}{m_{1} ! m_{2} !\left(2 t_{1}\right) !\left(2 t_{2}\right) !} \\
& \leq \frac{d ! 2^{2\left(d-m_{1}-m_{2}\right)}}{m_{1} ! m_{2} !\left(d-m_{1}-m_{2}\right) !}
\end{aligned}
$$

where $\sum^{\prime \prime}$ denotes the summation orer all $s_{1}$ and $s_{2}$ such that $s_{1}+s_{2}=d$, $s_{1}-\left|p^{1}-q^{1}\right|$ and $s_{2}-\left|p^{2}-q^{2}\right|$ are non-negative and even, and $t_{i}=\left(s_{i}-\mid p^{i}\right.$ $\left.-q^{i} \mid\right) / 2, m_{i}=\left|p^{i}-q^{i}\right|, \quad i=1,2$.

Apply the estimation (A.7) to (A.6) and then use the Stirling formula. Tedious calculations lead us to the estimation that the numerator of the right hand side of (A. 4) is less than $\exp n\left[8 e^{-1} A(\beta)(1+a\right.$ $\left.+\theta)+(2+2 a)(\delta(\beta)-\beta)+2 \log \left\{\left(1+a+\frac{\theta}{2}\right)\left(1+\frac{1}{a}+\frac{\theta}{2 a}\right)^{a}\right\}+o(1)\right]$, where $A(\beta)=\exp (-\beta+\delta(\beta))$. On the other hand, we can see easily that the denominator of that of (A.4) is greater than

$$
\text { Const. } \times 2^{(2+2 a) n} e^{-(\beta \delta(\beta))(2+2 a) n} / \sqrt{ } n .
$$


Thus we obtain

$$
\begin{aligned}
& P_{n}^{\omega}\left(\lambda_{n}(\sigma, \omega) \cap W_{n} \neq \phi\right) \\
& \quad \leq \exp n\left\{8 e^{-1} A(\beta)(1+a+\theta)+4(1+a) \delta(\beta)\right. \\
& \quad+2 \log F(\theta, a)+o(1)\}+o(1)
\end{aligned}
$$

as $n \rightarrow \infty$, where $F(\theta, a)=\left(1+a+\frac{\theta}{2}\right)\left(1+\frac{1}{a}+\frac{\theta}{2 a}\right)^{a} 2^{-(1+a)}$. It is easy to see that there exists $\theta_{a}>0$ such that $F(\theta, a)<1$ for $0 \leq \theta<\theta_{a}$. Since $A(\beta) \rightarrow 0$ and $\delta(\beta) \rightarrow 0$ as $\beta \rightarrow \infty$, we obtain

$$
\lim _{n \rightarrow \infty} P_{n}^{\omega}\left(\lambda_{n}(\sigma, \omega) \cap W_{n} \neq \phi\right)=0
$$

for sufficiently large $\beta>0$.

Lemma A.2. Let $Y=\left\{p=\left(p^{1}+\frac{1}{2}, p^{1}+\frac{1}{2}\right) \in L ; p^{1}>0\right\}$. We have (A. 8) $\quad \lim _{n \rightarrow \infty} P_{n}^{\omega}\left(\lambda_{n}(\sigma, \omega) \cap Y \neq \phi\right)=0$.

Proof. Divide $Y$ into two parts $Y_{n}^{1}=\left\{\left(p^{1}+\frac{1}{2}, p^{1}+\frac{1}{2}\right) \in Y ;-a n \leq p^{1}\right.$ $\leq 0\}$ and $Y_{n}^{2}=\left\{\left(p^{1}+\frac{1}{2}, p^{1}+\frac{1}{2}\right) \in Y ; p^{1}<-a n\right\}$. Applying the same technique as in the proof of Lemma A. 1, and noting that

$$
\begin{array}{r}
\frac{1}{\left(a n+p^{1}\right) !\left(n-p^{1}\right) !}=\left(\begin{array}{c}
a n+n \\
a n+p^{1}
\end{array}\right) \frac{1}{(a n+n) !} \leq \frac{1}{(a n) ! n !}, \\
\text { for } 0<a<1, p^{1} \leq 0 .
\end{array}
$$

we obtain

(A. 9) $P_{n}^{\omega}\left(\lambda_{n}(\sigma, \omega) \cap Y_{n}^{1} \neq \phi\right)$

$$
\begin{aligned}
& \leq \exp n\left\{8 e^{-1} A(\beta)(1+a+\theta)+4(1+a) \delta(\beta)+2 \log G(\theta, a)+o(1)\right\} \\
& \quad+o(1)
\end{aligned}
$$

as $n \rightarrow \infty$, where $G(\theta, a)=(1+a+\theta)\left(1+\frac{1+\theta}{a}\right)^{a} 2^{-(1+a)}$.

In the same way as above, we also obtain

(A. 10) $P_{n}^{\omega}\left(\lambda_{n}(\sigma, \omega) \cap Y_{n}^{2} \neq \phi\right)$

$$
\leq \exp n\left\{8 e^{-1} A(\beta)\left(1+a+\frac{3}{2} \theta\right)+2 e^{-1}\left(1+\frac{5 \theta}{4(1+a)}\right)\right.
$$




$$
\begin{aligned}
& \times\left(1+a+\frac{3}{2} \theta\right) A(\beta)^{2}+2(1+a)\left(\log \left(1+\frac{5 \theta}{4(1+a)}\right)\right. \\
- & \log 2+2 \delta(\beta))+o(1)\}+o(1),
\end{aligned}
$$

as $n \rightarrow \infty$. For sufficiently small $\theta$ and large $\beta$, the right hand sides of both (A.9) and (A. 10) converge to 0 as $u \rightarrow \infty$. This implies (A.8).

Norv it follows from Lemmas A. 1 and A. 2 that

$$
P_{n}^{\omega}\left(\left\{\sigma \in \Omega_{n} ; R_{A_{n}(\sigma, \omega)}(x) \text { touches the plus boundary }\right\}\right) \rightarrow 0
$$

as $n \rightarrow \infty$ for sufficiently large $\beta>0$ and any fixed $x \in Z^{2}$. This implies the convergence (A. 1) as in the proof of Theorem 1.

\section{References}

[1] Dobrushin, R, L., Gibbs state describing co-existence of phases for a three-dimensional Ising model, Theor. Probability Appl., 17 (1972), 582-600.

[2] Gallavotti, G., The phase separation line in the two-dimensional Ising model, Comm. Math. Phys., 27 (1672), 103-136.

[3] Gallavotti, G. and Miracle-Sole, S., Equilibrium states of the Ising model in the two phase region, Phys. Rev., 5B (1972), 2555-2559.

[4] Gallavotti, G. and Martin-Löf, A., Surface tension in the Ising model, Comm. Math. Phys., 25 (1972), 87-126.

[5] Lebowitz, J. L. and Martin-Löf, A., On the uniqueness of the equilibrium state for Ising spin systems, Comm. Math. Phys., 25 (1972), 276-282.

[6] Messager, A. and Miracle-Sole, S., Equilibrium state of the two-dimensional Ising model in the two phase region, Comm. Math. Phys., 40 (1975), 187-196.

[7] Messager, A. and Miracle-Sole, S., Report in the meeting on the infinite particle systems in Oberwolfach, Oct. 1976.

[8] Abraham, D. B., Odd operators and spinor algebras in lattice statistics. (To appear)

[9] Abraham, D. B. and Reed, P., Interface profile of the Ising ferromagnet in two dimensions, Comm. Math. Phys., 49 (1976), 35-46.

[10] Fortuin, C. M., Kasteleyn, P. W. and Ginibre, J., Correlation inequalities on some partially ordered sets, Comm. Math. Phys., 22 (1971), 89-103. 
